\title{
X-SHOOTER OBSERVATIONS OF MAIN-SEQUENCE STARS IN THE GLOBULAR CLUSTER NGC 2808: FIRST CHEMICAL TAGGING OF A He-NORMAL AND A He-RICH DWARF*
}

\author{
A. Bragaglia ${ }^{1}$, E. Carretta ${ }^{1}$, R. G. Gratton ${ }^{2}$, S. Lucatello ${ }^{2,3,4}$, A. Milone ${ }^{5}$, G. Piotto ${ }^{5}$, V. D’Orazi ${ }^{2}$, S. Cassisi ${ }^{6}$, \\ C. SNEDEN $^{7}$, AND L. R. BEDIN ${ }^{8}$ \\ ${ }^{1}$ INAF, Osservatorio Astronomico di Bologna, via Ranzani 1, 40127 Bologna, Italy; angela.bragaglia@ oabo.inaf.it, eugenio.carretta@ oabo.inaf.it \\ 2 INAF, Osservatorio Astronomico di Padova, vicolo dell'Osservatorio 5, 35122 Padova, Italy; raffaele.gratton@oapd.inaf.it, sara.lucatello@oapd.inaf.it, \\ valentina.dorazi@oapd.inaf.it \\ ${ }^{3}$ Excellence Cluster Universe, Technische Universität München, Boltzmannstr. 2, D-85748 Garching, Germany \\ ${ }^{4}$ Max-Planck-Institut für Astrophysik, D-85741 Garching, Germany \\ ${ }^{5}$ Dipartimento di Astronomia, Università di Padova, Vicolo dell’Osservatorio 2, I-35122 Padova, Italy; antonino.milone@ unipd.it, giampaolo.piotto@ unipd.it \\ ${ }^{6}$ INAF, Osservatorio Astronomico di Collurania, via M. Maggini, I-64100 Teramo, Italy; cassisi@ oa-teramo.inaf.it \\ ${ }^{7}$ Department of Astronomy and McDonald Observatory, The University of Texas, Austin, TX 78712, USA; chris@ verdi.as.utexas.edu \\ ${ }^{8}$ Space Telescope Science Institute, 2700 San Martin Drive, Baltimore, MD 21218, USA \\ Received 2010 July 1; accepted 2010 July 1; published 2010 August 11
}

\begin{abstract}
We present the first chemical composition study of two unevolved stars in the globular cluster NGC 2808, obtained with the X-shooter spectrograph at VLT. NGC 2808 shows three discrete, well-separated main sequences. The most accepted explanation for this phenomenon is that their stars have different helium contents. We observed one star on the bluest main sequence (bMS, claimed to have a high helium content, $Y \sim 0.4$ ) and the other on the reddest main sequence (rMS, consistent with a canonical helium content, $Y=0.245$ ). We analyzed features of $\mathrm{NH}, \mathrm{CH}$, $\mathrm{Na}, \mathrm{Mg}, \mathrm{Al}$, and $\mathrm{Fe}$. While $\mathrm{Fe}, \mathrm{Ca}$, and other elements have the same abundances in the two stars, the bMS star shows a huge enhancement of $\mathrm{N}$, a depletion of $\mathrm{C}$, an enhancement of $\mathrm{Na}$ and $\mathrm{Al}$, and a small depletion of $\mathrm{Mg}$ with respect to the rMS star. This is exactly what is expected if stars on the bMS formed from the ejecta produced by an earlier stellar generation in the complete $\mathrm{CNO}$ and $\mathrm{MgAl}$ cycles whose main product is helium. The elemental abundance pattern differences in these two stars are consistent with the differences in the helium content suggested by the color-magnitude diagram positions of the stars.
\end{abstract}

Key words: globular clusters: general - globular clusters: individual (NGC 2808) - stars: abundances - stars: evolution - stars: Population II

Online-only material: color figures

\section{INTRODUCTION}

The main sequence (MS) of the globular cluster (GC) NGC 2808 shows a wide color distribution (D'Antona et al. 2005). Very accurate HST ACS photometry (Piotto et al. 2007) reveals that the MS splits into three sequences that are likely formed in discrete episodes of star formation, slightly separated in age, and with different initial chemical composition. The MS of the massive, multi-metallicity GC $\omega$ Cen is also split into two (Bedin et al. 2004) or even more (Bellini et al. 2010) separate branches. NGC 2808 and $\omega$ Cen are presently the only GCs where the MS is clearly separated into discrete sequences; see the review by Piotto (2009).

The main role in producing these broad MSs is probably played by helium (e.g., Norris 2004). From the analysis of stacked spectra of 17 stars in each sequence of $\omega$ Cen, Piotto et al. (2005) clearly showed that the blue main sequence (bMS) is more metal rich than the red MS (rMS). The most reasonable way to reconcile these observations with stellar evolutionary theory is to suppose that the bMS is populated by stars born with larger helium abundance than the rMS stars. By analogy, He variations provide the simplest explanation for the three distinct NGC 2808 MSs, since the separation cannot be explained easily by age and/or metallicity variations. The three MSs can be fit by theoretical models with $\mathrm{He}$ content ranging from a normal

\footnotetext{
* Based on data collected at the ESO telescopes under GTO program 084.D-0070.
}

$Y=0.24$ to an extreme $Y=0.38$ value (see Figure 2 in Piotto et al. 2007).

Helium abundances cannot be directly measured by spectroscopy, except for high temperature, highly evolved stars (e.g., Moehler \& Sweigart 2006; Villanova et al. 2009). Fortunately, spectroscopic investigations of GCs over several decades have found large star-to-star and cluster-to-cluster variations in other light-element abundances, which strongly indicate the existence of multi-populations. In all clusters studied so far ${ }^{9}$ large star-tostar variations in light elements $\mathrm{O}, \mathrm{Na}, \mathrm{Mg}, \mathrm{Al}$, and $\mathrm{Si}$ are present (e.g., Carretta et al. 2009b, 2009c; and see Gratton et al. 2004 for a recent review). The variations have distinctive patterns: $\mathrm{O}$ and $\mathrm{Mg}$ abundances are positively correlated and are anticorrelated with $\mathrm{Na}, \mathrm{Al}$, and $\mathrm{Si}$ abundances. Such patterns leave little doubt about the chief nucleosynthesis culprit: high-temperature hydrogen fusion that includes $\mathrm{CNO}, \mathrm{NeNa}$, and $\mathrm{MgAl}$ cycles. Moreover, $\mathrm{Na}-\mathrm{O}$ and $\mathrm{Mg}-\mathrm{Al}$ anticorrelations have been found among unevolved MS and subgiant stars (Gratton et al. 2001), arguing that this pattern is produced by the ejecta of a first generation of now extinct more massive stars (Denisenkov \& Denisenkova 1989; Langer et al. 1993). The site of the H burning is still unclear. It might have occurred either in intermediatemass asymptotic giant branch (AGB) stars (D'Antona et al. 2002) or in fast-rotating massive stars on the MS (Decressin et al. 2007).

\footnotetext{
9 Possible exceptions are the poorly studied GCs Ter 7 and Pal 12 (Sbordone et al. 2005; Cohen 2004), where however only seven and four stars were studied, respectively.
} 


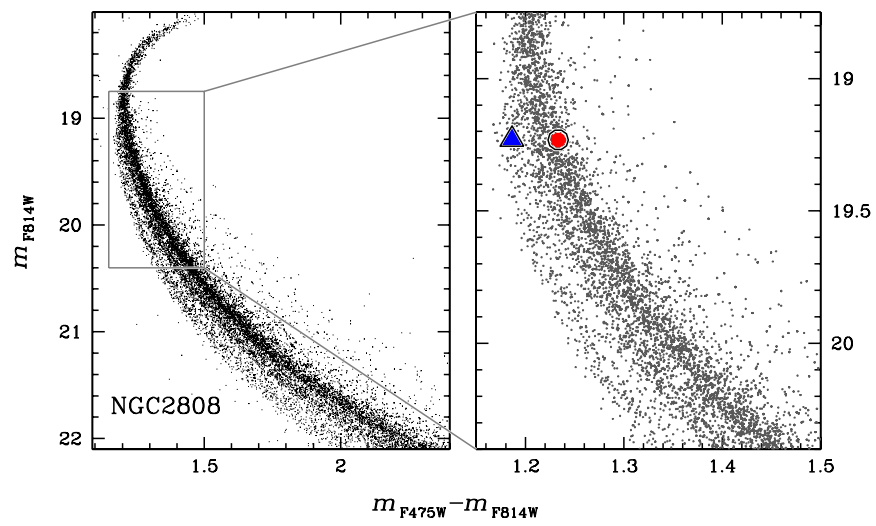

Figure 1. Triple MS of NGC 2808 observed with HST/ACS, corrected for differential reddening (Piotto et al. 2007). Our targets are indicated with a large circle (rMS star) and a large triangle (bMS star) in the enlargement (right panel).

(A color version of this figure is available in the online journal.)

The main outcome of $\mathrm{H}$ burning, helium, is expected to be directly related to the observed chemical pattern of light elements in GCs. Stars on the MS that are highly enriched in $\mathrm{He}$ should have large depletions of $\mathrm{O}$ and $\mathrm{Mg}$ and large enhancements of $\mathrm{Na}$ and $\mathrm{Al}$ (possibly $\mathrm{Si}$ as well). They should also populate the extreme blue horizontal branch (HB; see Bragaglia 2010 and Gratton et al. 2010) and the bluer MS.

NGC 2808 is the ideal target to derive a clear-cut confirmation of these effects. The close link between He enhancement and the simultaneous depletion/increase in light elements may be investigated in this GC because (1) the three distinct MSs allow unambiguous selection of stars with likely different He contents; (2) this cluster has an unusual HB, strongly bimodal (or even trimodal), and with a long blue (hot) tail (e.g., Bedin et al. 2000) that has been connected to different He enrichments (D'Antona \& Caloi 2004); and (3) it shows a very extended $\mathrm{Na}-\mathrm{O}$ anticorrelation (Carretta et al. 2006), also implying very large $\mathrm{He}$ enhancements (see also Bragaglia et al. 2010). As discussed by Piotto et al. (2007), there seems to be a clear connection between the various groups of MS, red giant branch (RGB), and HB stars, according to star counts in the studies of Piotto et al. (2007), Carretta et al. (2006), and Bedin et al. (2000), respectively.

With the advent of X-shooter (Vernet et al. 2009) at the Very Large Telescope (VLT), medium-resolution spectra useful for abundance analysis are currently within reach for faint MS stars of NGC 2808. In this Letter, we present the first results of the chemical tagging of two stars observed in situ on the bluest and the red MS of this cluster.

\section{OBSERVATION, REDUCTION, AND ANALYSIS}

We selected our targets among the brightest stars for which the separation of the MSs is still possible, since the three MSs merge near the turnoff of the cluster (Figure 1). As confirmed on theoretical grounds (Salaris \& Cassisi 2005), the combined effects of larger brightness and shorter lifetime during the MS stage when the initial He content increases largely cancel out. As a consequence, two isochrones of same age and different He contents almost overlap in the turnoff and subgiant regions. From the ACS photometric catalog (Piotto et al. 2007), we selected two MS stars in NGC 2808 that (1) have proper motions typical of cluster stars, (2) have a probability $>85 \%$ of being on the bMS and $>95 \%$ of being on the rMS, based
Table 1

Information on the Two Targets and Derived Abundances

\begin{tabular}{lcc}
\hline \hline \multicolumn{1}{c}{ Star } & rMS Star & bMS Star \\
\hline R.A. (h:m:s) & $09: 11: 36.29$ & $09: 11: 30.80$ \\
Decl. (d:p:s) & $-64: 55: 19.17$ & $-64: 53: 22.49$ \\
$m_{\text {F475W }}(\mathrm{mag})$ & 20.465 & 20.416 \\
$m_{\mathrm{F} 814 \mathrm{~W}}(\mathrm{mag})$ & 19.232 & 19.230 \\
$T_{\text {eff }}(\mathrm{K})$ & 6252 & 6479 \\
$\log g$ & 4.32 & 4.27 \\
{$[\mathrm{C} / \mathrm{Fe}]$} & -0.3 & -0.7 \\
{$[\mathrm{~N} / \mathrm{Fe}]$} & +0.5 & +2.0 \\
{$[\mathrm{Na} / \mathrm{Fe}]$} & -0.3 & +0.8 \\
{$[\mathrm{Mg} / \mathrm{Fe}]$} & +0.4 & +0.1 \\
{$[\mathrm{Al} / \mathrm{Fe}]$} & -0.2 & +1.1
\end{tabular}

Notes. For both stars, we used $[\mathrm{Fe} / \mathrm{H}]=-1.1$ and $v_{t}=0.8 \mathrm{~km} \mathrm{~s}^{-1}$. The adopted distance modulus and reddening are $(m-M)_{V}=15.0$ and $E(B-V)=0.18$, respectively.

on their magnitude and colors, respectively, and (3) do not have neighbors closer than $1^{\prime \prime} .5$.

As part of the Italian X-shooter guaranteed time observations (GTO), we observed these two MS stars with the X-shooter spectrograph at VLT-UT2 on 2010 January 22-23. The wavelength coverage of $\mathrm{X}$-shooter ranges from the atmospheric UV limit to the near-infrared. For our observations, the slit width was set at $1^{\prime \prime}$ or 0.8 , but the resolution $(R \sim 10,000)$ was mostly dictated by the sub-arcsecond seeing, especially for the second night. We took four and five $1 \mathrm{hr}$ exposures for star 5 and 6 , respectively. Information on the targets is given in Table 1 and their position in the color-magnitude diagram (CMD) is shown in Figure 1. The observations were optimized for the UV and visual part of the spectrum; i.e., we did not use the nod option, essential to subtract the sky in IR. Therefore, we use here mostly the UV/blue spectrum $(\sim 3300-5500 \AA)$ and part of the visible ( $\sim 500-10000 \AA)$. The spectra were reduced using the preliminary X-shooter pipeline (v0.9.4; see Goldoni et al. 2006) and standard IRAF ${ }^{10}$ routines. Each stellar exposure was bias-corrected and flat-fielded, calibrated in wavelength, then extracted and corrected for sky background. Our targets are near the limit of the instrument response, and the sky level is comparable to the star signal (or higher than it, in the presence of even a small Moon contribution). The individual spectra have signal-to-noise ratio $(\mathrm{S} / \mathrm{N})$ from about 10 to about 20 . They were combined, weighting them with their $\mathrm{S} / \mathrm{N}$, and shifted to zero radial velocity. The radial velocities were measured using about 35 lines; the heliocentric values are 75 and $80 \mathrm{~km} \mathrm{~s}^{-1}$ (rms $10 \mathrm{~km} \mathrm{~s}^{-1}$ ), for the rMS star and the bMS star, respectively. Given the resolution and the uncertainty due to the use of a (not filled) slit, they well compare to the value of $93.6 \mathrm{~km} \mathrm{~s}^{-1}$ reported by Harris (1996).

To derive temperatures and gravities, we used a combination of photometric information and isochrone fitting. Isochrones (Pietrinferni et al. 2006), distance modulus, and reddening are those used in Piotto et al. (2007) to fit the discrete MSs observed in NGC 2808. The isochrone consistent with the rMS position of the first star has a canonical He abundance $(Y=0.248)$ and that for the bMS position of the second star has enhanced He $(Y \simeq 0.40)$. In the current interpretation, rMS star is of first generation and bMS star of second generation. The individual

\footnotetext{
10 IRAF is distributed by the National Optical Astronomical Observatory, which is operated by the Association of Universities for Research in Astronomy, under contract with the National Science Foundation.
} 

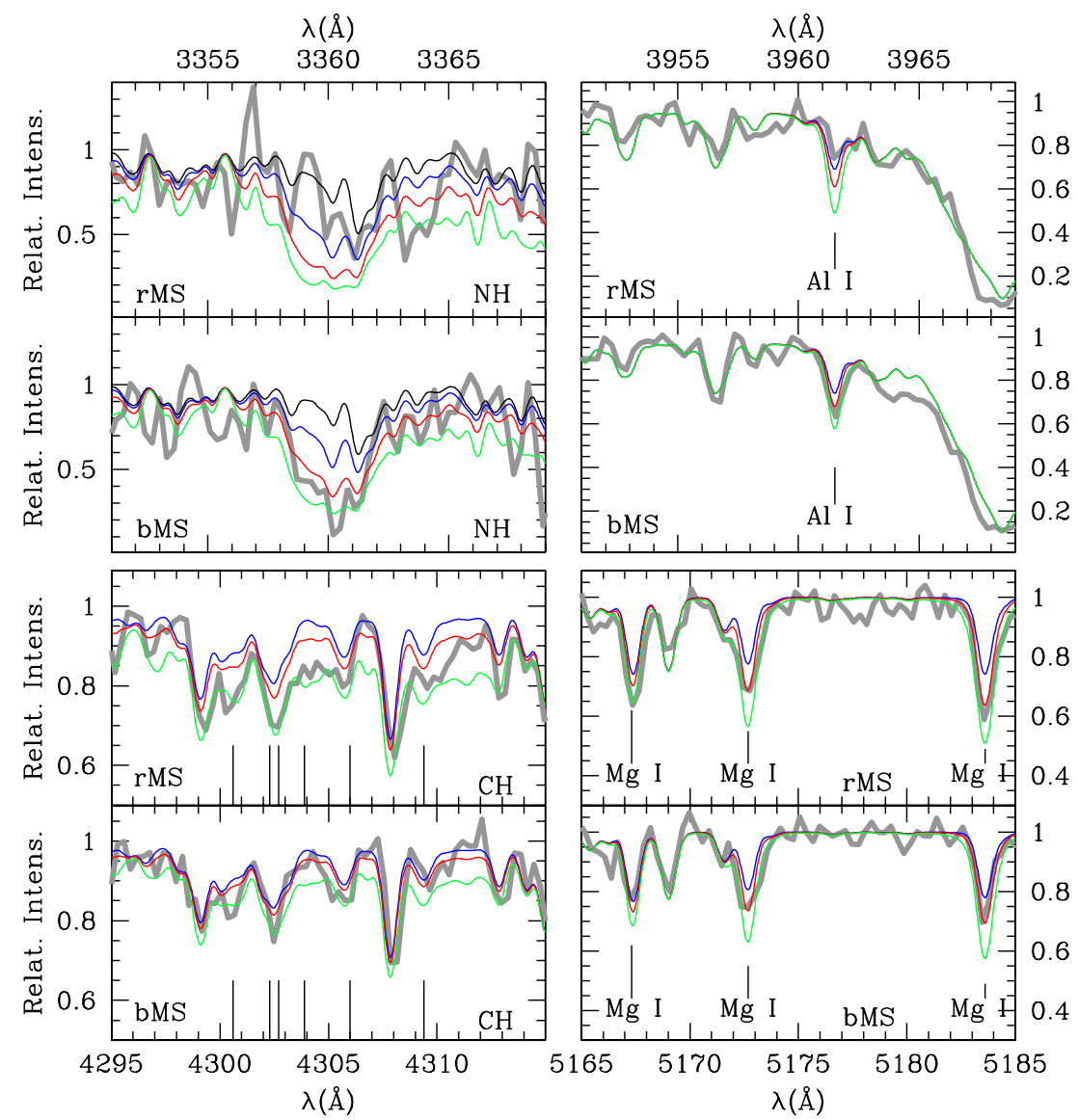

Figure 2. Synthetic (light lines) and observed spectra (heavier, gray lines) for rMS star (upper panels) and bMS star (lower panels) for $\mathrm{NH}, \mathrm{Al}, \mathrm{Mg}$, and $\mathrm{CH}$, clockwise from the upper left panel. All spectra were normalized to unity. In all panels the vertical lines indicate the spectral lines synthesized. The different synthetic spectra were computed with the following abundances: $[\mathrm{N} / \mathrm{Fe}]=0,1.0,1.5,2.0 ;[\mathrm{Al} / \mathrm{Fe}]=-0.7,-0.2,0.3,0.8(\mathrm{LTE}) ; \mathrm{Mg} / \mathrm{Fe}]=-0.5,0.0,0.5 ;$ and $[\mathrm{C} / \mathrm{Fe}]=-1.0$, $-0.5,0.0$

(A color version of this figure is available in the online journal.)

values for $T_{\text {eff }}$ and $\log g$ are indicated in Table 1. For both stars, we adopted the NGC 2808 metallicity derived from highresolution spectra by Carretta et al. (2006), $[\mathrm{Fe} / \mathrm{H}]=-1.1$, and assumed a microturbulent velocity $v_{t}=0.8 \mathrm{~km} \mathrm{~s}^{-1}$. These are reasonable assumptions, given the identical evolutionary states and the internal homogeneity in heavy elements and metallicity. ${ }^{11}$ The exact value of $v_{t}$ does not strongly influence the results presented here.

The main interest of our analysis lies in the differential analysis of the two stars. However, the temperatures adopted are supported by the Balmer lines, and we confirmed the assumed metallicity by computing synthetic spectra near a few Fe I lines with good $g f$ values. We did not explicitly took into account an enhancement in He in the stellar atmospheres; however, the expected effect on iron abundance determination is negligible (see discussions in Carretta et al. 2006; Bragaglia et al. 2010) and this is most probably true for the other elements. Although we did not do a detailed analysis of other heavy-element species, we noted that lines of, e.g., Ti and Ca have approximately the same strength in both stars. None of the suggested pollution sources that contribute hydrogen-burning products to newly forming stars should contribute elements beyond Si. Heavier $\alpha$ or Fepeak abundances should be the same in all NGC 2808 stars, and our spectra do not contradict this expectation. 11 With very few exceptions, the stars metallicity is very homogeneous in GCs
(better than 10\%; Carretta et al. 2009a).

\section{RESULTS}

Adopting the stellar parameters defined above, we used standard routines to compute synthetic spectra for some particularly interesting elements: $\mathrm{N}$ (from the $\mathrm{NH}$ feature at $3360 \AA$ ), $\mathrm{Al}$ (from the $3961 \AA$ resonant line), $\mathrm{Mg}$ (from the $\mathrm{Mg} \mathrm{b}$ lines near $5180 \AA$ ), $\mathrm{C}$ (from the $\mathrm{CH}$ features in the $G$ band near $4300 \AA$ ), and $\mathrm{Na}$ (from the 8183-94 ̊ doublet). The spectral syntheses shown in the figures were computed with the LTE spectroscopic analysis code ROSA (Gratton 1988) for the atomic lines (Al, Mg, and Na) and MOOG (Sneden 1973) for the molecules $(\mathrm{NH}, \mathrm{CH})$. However, all the synthesis work was checked independently using both codes; results are in very good agreement.

Abundances for all elements are presented in Table 1. The derived values have conservative error estimates (mostly due to the uncertain continuum placement) of $0.1 \mathrm{dex}$ for $\mathrm{Na}$ (and $\mathrm{Fe}$ ), and 0.2 dex for $\mathrm{N}, \mathrm{C}, \mathrm{Mg}$, and $\mathrm{Al}$. We stress however that the main result of our analysis lies in the difference between the abundance patterns of the two stars, more than in the absolute values for the chemical abundances. We will show that the lightelement differences between rMS star and bMS star exceed their uncertainties.

Comparison of observed and synthetic spectra are shown in Figure 2 for $\mathrm{NH}, \mathrm{Al}, \mathrm{CH}$, and $\mathrm{Mg}$ I features. From the closest observed/synthetic matches, we estimated the abundances that are given in Table 1. The expectations are that $\mathrm{N}$ and $\mathrm{Al}$ should be increased and C and Mg should be decreased in bMS star 


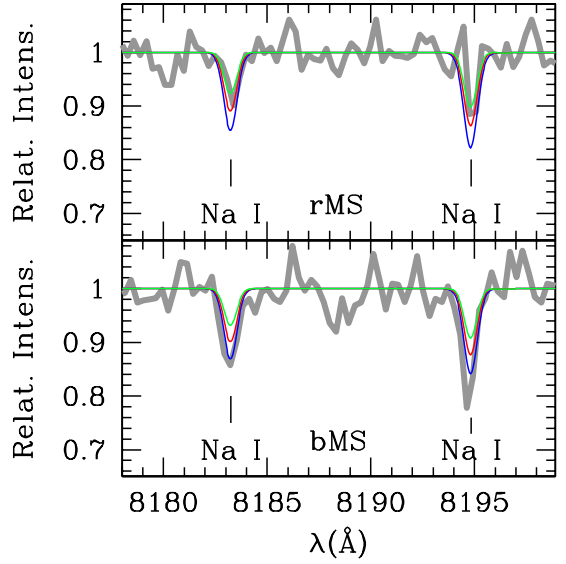

Figure 3. Observed (gray, thick lines) and synthetic (thin lines) spectra for the $\mathrm{Na}$ features at $8183-8194 \AA$. The synthetic spectra are for $[\mathrm{Na} / \mathrm{Fe}]=0.0,0.4$., and 0.8 (LTE).

(A color version of this figure is available in the online journal.)

with respect to the values for rMS star (the one of supposedly normal, primordial composition), following what has been found for evolved RGB stars (e.g., Ivans et al. 2001; Cohen et al. 2002; Ramírez \& Cohen 2003; Carretta et al. 2009b). Figure 2 demonstrates that the two stars have different lightelement spectra. This is most obvious for $\mathrm{N}$ : the $\mathrm{NH}$ absorption is much stronger in bMS star than in rMS star. Neither details in the spectrum normalization nor (small) differences in the atmospheric parameters can account for this difference.

The Al abundance was derived only from the $3961 \AA$ resonance line, since its doublet partner at $3944 \AA$ is a blend (Arpigny $\&$ Magain 1983). The synthesis was computed adopting the Ca abundance appropriate for NGC $2808([\mathrm{Ca} / \mathrm{Fe}]=+0.34$; Carretta et al. 2009b) to reproduce the Ca II $\mathrm{H}$ and $\mathrm{K}$ lines. The values for $\mathrm{Al}$ and $\mathrm{Mg}$ given in Table 1 are corrected for NLTE effects according to Gehren et al. (2004); the corrections are about +0.5 dex for $\mathrm{Al}$ and +0.06 dex for $\mathrm{Mg}$, respectively, for both stars.

The huge abundance of $\mathrm{N}$ found for bMS star (which also had decreased C) can be explained only with the transformation of (virtually) all oxygen into nitrogen. Our findings seem to indicate that we are seeing, in the gas from which this star formed, the outcome of the complete CNO cycle. Actually, if we combine the $[\mathrm{C} / \mathrm{Fe}]$ and $[\mathrm{N} / \mathrm{Fe}]$ values of Table 1 with the solar C, N, and O abundances by Asplund et al. (2009) and with the maximum $[\mathrm{O} / \mathrm{Fe}]$ ratio for RGB stars in NGC 2808 (Carretta et al. 2006), even large depletions of $\mathrm{O}([\mathrm{O} / \mathrm{Fe}]<-1)$ cannot reproduce a constant sum of the CNO elements. This would be reproduced by assuming $[\mathrm{N} / \mathrm{Fe}] \sim 1.4$ for bMS star. We note that a systematic offset of $\sim 0.6$ dex in $\mathrm{N}$ abundances from our analysis would produce a roughly solar scaled $[\mathrm{N} / \mathrm{Fe}]$ ratio for rMS star, which would agree fairly well with the values usually assumed for field halo stars (Gratton et al. 2000). Once again, what is most important is the difference between the derived abundances and this is a sound result.

Unfortunately, it was not possible to measure $\mathrm{O}$ abundances for these two stars, since the O triplet at 7771-7774 $\AA$ is weak and falls in a wavelength region where the sky subtraction is difficult for these very faint objects. However, we were able to estimate the $\mathrm{Na}$ abundances. Figure 3 shows observed and synthetic spectra surrounding the 8183-8194 $\AA \mathrm{Na}$ I lines. We see in the figure that the $\mathrm{Na}$ lines are stronger in bMS star than in rMS star and this is reflected in the abundance ratios indicated

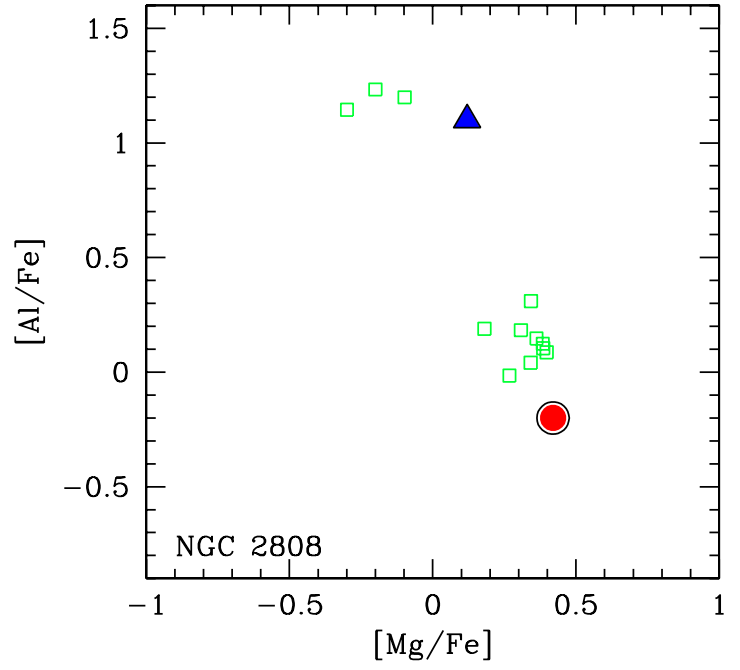

Figure 4. Anticorrelation between $\mathrm{Al}$ and $\mathrm{Mg}$ abundances. The open squares are for 12 RGB stars analyzed in Carretta et al. (2009b), while the filled circle and triangle indicate the values for rMS star and bMS star, respectively.

(A color version of this figure is available in the online journal.)

in Table 1. These abundances include NLTE corrections (about -0.1 dex) as recommended by Gratton et al. (1999). The chief limitation is the strong telluric-line contamination in this spectral region. The tellurics were eliminated by division of the program star spectra with that of a hot, rapidly rotating star, using an IRAF routine for this task. The cleaning quality is much better for the bluest of the two lines, so that our $\mathrm{Na}$ abundances rest on that single feature. They are qualitatively confirmed by the other line and by the relative strengths of the $\mathrm{Na} \mathrm{D}$ lines. Unfortunately, strong interstellar absorption and sky emission made the derivation of $\mathrm{Na}$ abundance from the $\mathrm{D}$ lines less secure, given the radial velocity at the time of observation and the moderate resolution of the X-shooter spectra.

The present $\mathrm{Al}$ and $\mathrm{Mg}$ results are in good agreement with those found from high resolution UVES spectra of 12 red giants in NGC 2808 by Carretta et al. (2009b). In Figure 4, we plot the $\mathrm{Mg}-\mathrm{Al}$ anticorrelation for the RGB stars and the two MS stars analyzed here. Apart from a possible small zero-point effect due to the use of different lines, corrections for NLTE, etc., the two MS stars do nicely participate in the same trend defined by the giants. The rMS and the bMS star fall in the Mg-rich/ Al-poor and Mg-poor/Al-rich groups, respectively. This result indicates that the extreme abundance pattern of the Al-rich, $\mathrm{Mg}$ poor stars in NGC 2808 must have been produced by pollution from a previous stellar generation in the clusters. Deep mixing has been recently revisited as an explanation for the extreme chemical abundances of RGB stars in GCs (see, e.g., D'Antona \& Ventura 2007; Lee 2010). However, since deep mixing cannot have been responsible for the light-element abundances in the bMS star, it is unlikely to have caused the identical pattern in evolved RGB stars of NGC 2808.

Finally, it is interesting to note that the $\mathrm{Ba}$ abundance, as indicated by the resonance Ba line at $4554 \AA$, seems the same for the two MS stars. If confirmed by more quantitative analysis, this would probably exclude a significant contribution from lowmass AGB stars (e.g., Yong et al. 2009) to the pool of gas from which the second-generation stars originated. The consequence is that the handful of stars observed to have strong enhancement in $\mathrm{Ba}$ and other $s$-process elements in some GCs must have another origin, likely from mass transfer from a former AGB 
companion in a binary system (see V. D'Orazi et al. 2010, in preparation).

\section{CONCLUSIONS}

In conclusion, the chemical pattern we found from the first abundance analysis of a star on the He-rich MS of NGC 2808 is exactly what is expected if stars on the bMS formed from ejecta produced by an early stellar generation via protoncapture reactions in $\mathrm{H}$ burning at high temperature, accompanied the main outcome of this nuclear burning, i.e., helium. The extreme $\mathrm{Al}$ enhancement and $\mathrm{Mg}$ depletion observed in the bMS star argues against a deep mixing hypothesis for the extreme chemical abundances of RGB stars in GCs.

Observations of a larger sample of unevolved stars in this cluster and others with suspected He variations would be welcome.

We thank Paolo Molaro for the nice observations made on behalf of the Italian X-shooter GTO team, and Valentina D'Odorico for help and suggestions with the data reduction. The organizing work of Paul Groot and Sofia Randich is acknowledged. Funding come from PRIN-MIUR 2007, the Italian GTO X-shooter Consortium, and US National Science Foundation grant AST-0908978. This Letter is dedicated to Roberto Pallavicini, late Italian PI of the X-shooter Consortium, who spent a lot of time and work for the realization of this fine instrument.

\section{REFERENCES}

Arpigny, C., \& Magain, P. 1983, A\&A, 127, L7

Asplund, M., Grevesse, N., Sauval, A. J., \& Scott, P. 2009, ARA\&A, 47, 481

Bedin, L. R., Piotto, G., Anderson, J., Cassisi, S., King, I. R., Momany, Y., \& Carraro, G. 2004, ApJ, 605, L125

Bedin, L. R., Piotto, G., Zoccali, M., Stetson, P. B., Saviane, I., Cassisi, S., \& Bono, G. 2000, A\&A, 363, 159

Bellini, A., Bedin, L. R., Piotti, G., Milone, A. P., Marino, A. F., \& Villanova, S. 2010, AJ, in press (arXiv:1006.4157)

Bragaglia, A. 2010, in IAU Symp. 268, Light elements in the Universe, ed. C. Charbonnel et al. (Cambridge: Cambridge Univ. Press), 119

Bragaglia, A., Carretta, E., Gratton, R., D’Orazi, V., Cassisi, S., \& Lucatello, S. 2010, A\&A, in press (arXiv:1005.2659)

Carretta, E., Bragaglia, A., Gratton, R. G., D'Orazi, V., \& Lucatello, S. 2009a, A\&A, 508, 695
Carretta, E., Bragaglia, A., Gratton, R. G., Leone, F., Recio-Blanco, A., \& Lucatello, S. 2006, A\&A, 450, 523

Carretta, E., Bragaglia, A., Gratton, R. G., \& Lucatello, S. 2009b, A\&A, 505, 139

Carretta, E., et al. 2009c, A\&A, 505, 117

Cohen, J. G. 2004, AJ, 127, 1545

Cohen, J. G., Briley, M. M., \& Stetson, P. B. 2002, AJ, 123, 2525

D’Antona, F., Bellazzini, M., Caloi, V., Fusi Pecci, F., Galleti, S., \& Rood, R. T. 2005, ApJ, 631, 868

D'Antona, F., \& Caloi, V. 2004, ApJ, 611, 871

D’Antona, F., Caloi, V., Montalban, J., Ventura, P., \& Gratton, R. 2002, A\&A, 395, 69

D’Antona, F., \& Ventura, P. 2007, MNRAS, 379, 1431

Decressin, T., Meynet, G., Charbonnel, C., Prantzos, N., \& Ekstrom, S. 2007, A\&A, 464, 1029

Denisenkov, P. A., \& Denisenkova, S. N. 1989, A. Tsir., 1538, 11

Gehren, T., Liang, Y. C., Shi, J. R., Zhang, H. W., \& Zhao, G. 2004, A\&A, 413, 1045

Goldoni, P., Royer, F., François, P., Horrobin, M., Blanc, G., Vernet, J., Modigliani, A., \& Larsen, J. 2006, Proc. SPIE, 6269, 62692K

Gratton, R. G. 1988, Rome Obs. Preprint Ser., 29

Gratton, R. G., Carretta, E., Bragaglia, A., Lucatello, S., \& D’Orazi, V. 2010, A\&A, in press (arXiv: 1004.3682)

Gratton, R. G., Carretta, E., Eriksson, K., \& Gustafsson, B. 1999, A\&A, 350, 955

Gratton, R. G., Sneden, C., \& Carretta, E. 2004, ARA\&A, 42, 38

Gratton, R. G., Sneden, C., Carretta, E., \& Bragaglia, A. 2000, A\&A, 354, 169

Gratton, R. G., et al. 2001, A\&A, 369, 87

Harris, W. E. 1996, AJ, 112, 1487

Ivans, I. I., Kraft, R. P., Sneden, C., Smith, G. H., Rich, R. M., \& Shetrone, M. 2001, AJ, 122, 1438

Langer, G. E., Hoffman, R., \& Sneden, C. 1993, PASP, 105, 301

Lee, J.-W. 2010, MNRAS, 405, L36

Moehler, S., \& Sweigart, A. V. 2006, A\&A, 455, 943

Norris, J. E. 2004, ApJ, 612, L25

Pietrinferni, A., Cassisi, S., Salaris, M., \& Castelli, F. 2006, ApJ, 642, 797

Piotto, G. 2009, in IAU Symp. 258, The Ages of Stars, ed. E. E. Mamajek, D. R. Soderblom, \& R. F. G. Wyse (Cambridge: Cambridge Univ. Press), 233

Piotto, G., et al. 2005, ApJ, 621, 777

Piotto, G., et al. 2007, ApJ, 661, L53

Ramírez, S. V., \& Cohen, J. G. 2003, AJ, 125, 224

Salaris, M., \& Cassisi, S. 2005, Evolution of Stars and Stellar Populations (Weinheim: Wiley, ESO)

Sbordone, L., Bonifacio, P., Marconi, G., Buonanno, R., \& Zaggia, S. 2005, A\&A, 437, 905

Sneden, C. 1973, ApJ, 184, 839

Vernet, J., D’Odorico, S., Christensen, L., Dekker, H., Mason, E., Modigliani, A., \& Moehler, S. 2009, Messenger, 138, 4

Villanova, S., Piotto, G., \& Gratton, R. G. 2009, A\&A, 499, 755

Yong, D., Grundahl, F., D’ Antona, F., Karakas, A. I., Lattanzio, J. C., \& Norris, J. E. 2009, ApJ, 695, L62 